\title{
Male circumcision, human papillomavirus and cervical cancer: from evidence to intervention
}

\author{
F Xavier Bosch, Ginesa Albero, Xavier Castellsagué
}

\section{Introduction}

The relationship between male circumcision and genital human papillomavirus (HPV) infection and cervical cancer has been greatly clarified in recent years. Along with the strong evidence from both observational studies and three randomised clinical trials (RCTs) consistently showing that male circumcision reduces the risk of HIV infection, ${ }^{1-4}$ the rapidly accumulating evidence for a protective effect of male circumcision on HPV and cervical cancer has prompted the suggestion that male circumcision could be considered a major intervention measure to prevent the incidence of both diseases. Thus, the introduction of safe male circumcision, if widely accepted and implemented, might be in particular relevant in developing countries in which no other measures to curtail AIDS and cervical cancer are available.

This commentary examines the evidence on the protective effect of male circumcision on penile HPV through a meta-analysis on the association between male circumcision and penile HPV and also summarises the authors' own research data from a large multicentre casecontrol study that assessed the relationship between male circumcision and cervical cancer. Both pieces of evidence strongly suggest that male circumcision may indeed have an additional protective value against HPV and cervical cancer to the already established benefit for HIV prevention.

\section{Circumcision and penile HPV: an updated meta-analysis}

We have recently published results from a systematic review and meta-analysis of published studies reporting on the association between male circumcision and penile HPV or genital warts up to March 2006.5 Here we present an update that includes published studies on the topic up to September 2007. The details of the studies included in the first meta-analysis in terms of study design, type of study population, anatomical sites sampled, and the HPV DNA detection methods used have already been described. This update adds three additional studies ${ }^{6-8}$ that increase the number of subjects to a total of 5880

J Fam Plann Reprod Health Care 2009; 35(1): 5-7

${ }^{1}$ Catalan Institute of Oncology, IDIBELL; Cancer Epidemiology Research Programme (CERP); Unit of Infections and Cancer (UNIC); L'Hospitalet de Llobregat, Barcelona, Spain

${ }^{2}$ CIBER en Epiemiología y Salud Pública (CIBERESP), Spain 3Programme in Public Health and the Methodology of Biomedical Research, Universitat Autònoma de Barcelona (UAB), Cerdanyola del Vallès, Spain

F Xavier Bosch 1,2 , MD, PhD, Chief of International Affairs and Cancer Epidemiology Research Program (CERP)

Ginesa Albero $1,2,3$, MSc, Statistician

Xavier Castellsagué ${ }^{1}, \mathrm{MD}, \mathrm{PhD}$, Coordinator of Unit of Infections and Cancer (UNIC)

Correspondence to: Professor F Xavier Bosch, Unit of Infections and Cancer (UNIC), Cancer Epidemiology Research Programme (CERP), Catalan Institute of Oncology, IDIBELL, Avda. Gran Via, s/n Km 2.7, L'Hospitalet de Llobregat, Barcelona 08907, Spain. E-mail: x.bosch@iconcologia.net circumcised and 4257 uncircumcised men. ${ }^{9}$ These subjects were recruited in 14 studies conducted in the USA (five studies), Mexico (two studies), Australia (two studies), South Korea, Denmark, England, Kenya, and in a multinational study conducted in Brazil, Colombia, Spain, Thailand and The Philippines. Most studies were of cross-sectional nature. The study populations were very heterogeneous across studies and included university students, sexually transmitted infection (STI)/vasectomy clinic patients, military men, subjects from the general population, truck drivers or husbands of women with and without cervical cancer. Age at circumcision was only reported in two studies, thus its overall potential effect on penile HPV could not be appropriately assessed.

After taking into account the key potential sources of heterogeneity and publication bias, we found inverse associations between male circumcision and penile HPV and, to a lesser extent, genital warts. Thus, male circumcision was associated with a statistically significant reduced risk of penile HPV [odds ratio (OR) 0.52 , 95\% CI 0.33-0.82; Figure 1]. This inverse association was not as strong, but still statistically significant, when penile HPV and genital warts were combined into a single outcome variable (OR 0.63, 95\% CI 0.45-0.90). In contrast, the inverse association between male circumcision and genital warts did not reach statistical significance (OR 0.89 , 95\% CI $0.59-1.33)$. It is well established that genital warts are virtually always caused by HPV and that the diagnosis is highly accurate. The lack of a strong effect of male circumcision on genital warts might be due to the fact that in addition to the urethra, glans and prepuce, these lesions frequently occur in the penile shaft, a site for which circumcision is unlikely to exert an effect.

\section{Circumcision and cervical cancer}

A recent solid piece of evidence confirming the potential impact of male circumcision on HPV transmission and cervical carcinogenesis comes from the International Agency for Research on Cancer (IARC) multicentre casecontrol study on cervical cancer conducted in Spain,

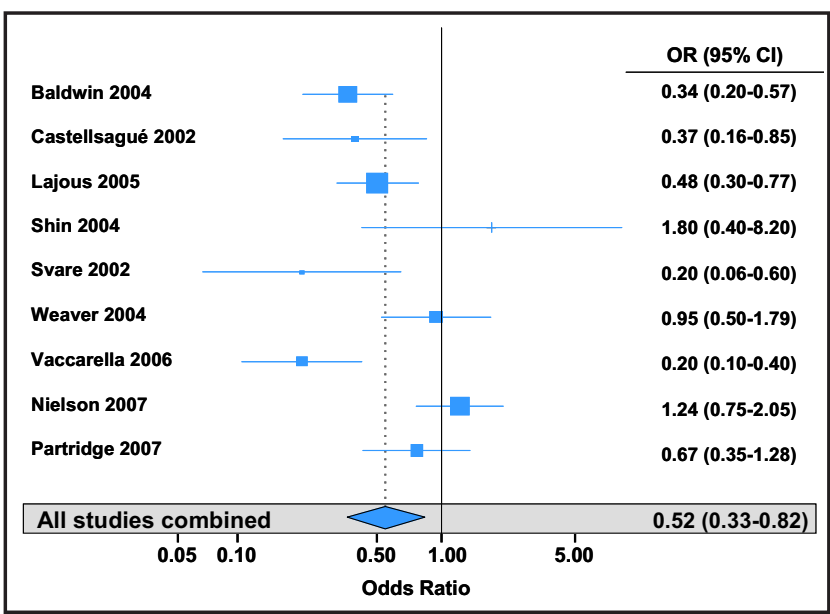

Figure 1 Meta-analysis for the association between male circumcision and penile human papillomavirus (HPV) DNA. Cl, confidence interval; OR, odds ratio 


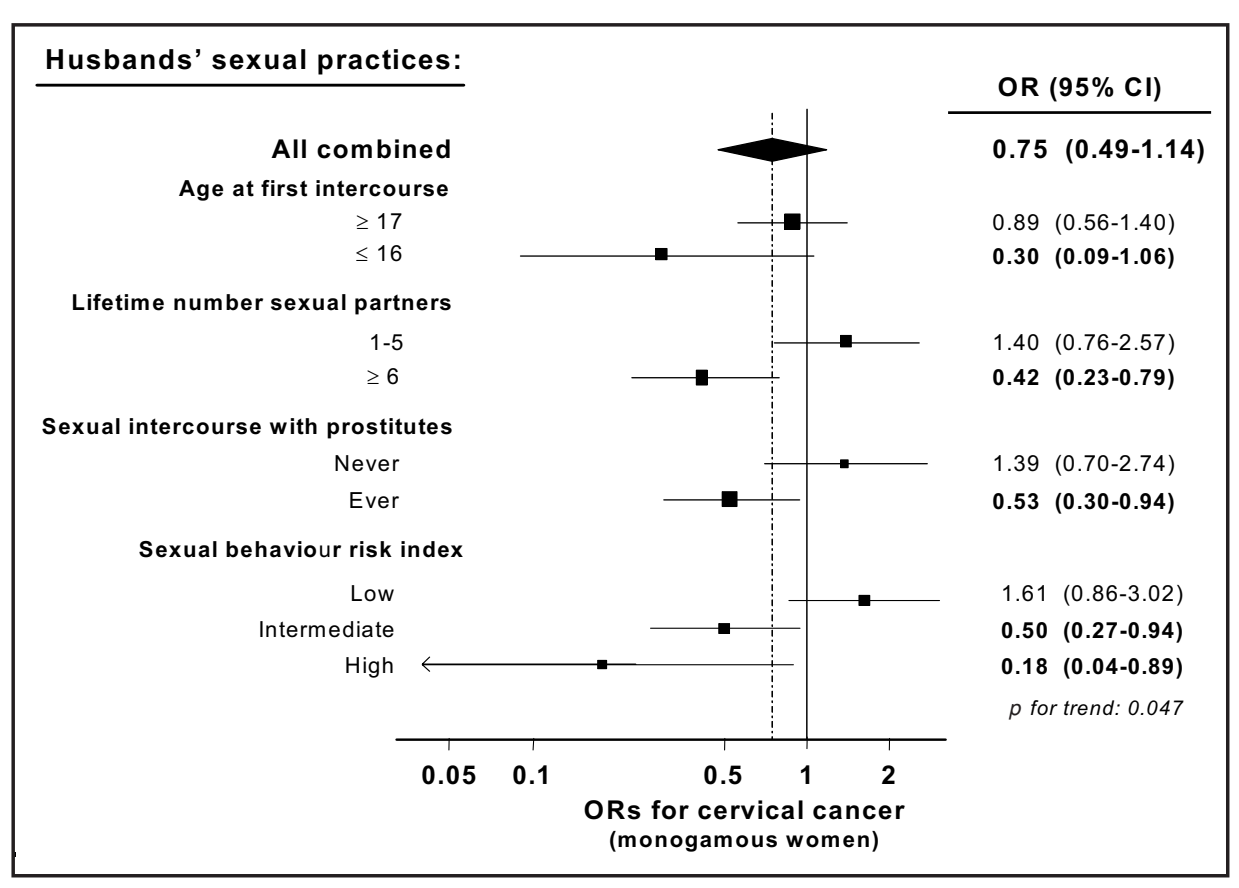

Figure 2 Odds ratios for the association between male circumcision and cervical cancer in monogamous women overall and according to husbands' sexual behaviour characteristics. The black square and horizontal line represents the odds ratio (OR) and 95\% confidence interval $(95 \% \mathrm{Cl})$ for that stratum. The area of the black squares is proportional to the estimate's precision. The diamond represents the combined OR and $95 \% \mathrm{Cl}$. Husbands with a high sexual behaviour risk index were those with sexual debut before 17 years of age and six or more lifetime number of sexual partners. Husbands with a low sexual behaviour risk index were those with a later sexual debut and a lower number of sexual partners. The remaining husbands were considered to have an intermediate sexual behaviour risk index. For all models reference group is wives of uncircumcised men in that stratum. Models were adjusted by study, male's and female's age, male's education attained, male's age at first sexual intercourse, male's frequency of genital washing after sex, male's lifetime number of sexual partners and female's age at first intercourse
Colombia, Brazil, Thailand and The Philippines. ${ }^{10}$ Female case patients were women who had newly diagnosed, histologically confirmed cervical carcinoma in situ or invasive cervical cancer. Control women were recruited from the general population in Spain and Colombia and from the same hospitals as the case patients in the other centres. Men who were eligible for the present study were the husbands or stable partners of the women with cervical cancer and the control women enrolled in each centre. Two samples of exfoliated cells were obtained from the penis: one from the distal urethra with the use of a very thin, wet, cotton-tipped swab and one from the external surface of the glans and coronal sulcus with the use of a standard size, wet, cotton-tipped swab. A total of 1913 couples were included in the final analysis. The main objective of the study was to compare penile HPV DNA detection in circumcised and uncircumcised men and to estimate a woman's risk of developing cervical HPV and cervical cancer according to the husband's circumcision status and sexual behaviour. Among the 370 circumcised men, $49.5 \%$ had the procedure before puberty (before 13 years of age) and $82 \%$ at or before sexual debut. The mean and median ages at circumcision were 15 and 13 years, respectively.

Penile HPV was detected in 166/847 uncircumcised men $(19.6 \%)$ and in 16/292 circumcised men (5.5\%). After adjustment for age at first intercourse, lifetime number of sexual partners, and other potential confounders, we found that circumcised men were about three times less likely to harbour HPV in their penis than did uncircumcised men (OR $0.37,95 \%$ CI $0.16-0.85$ ).

Consistent with the venereal nature of HPV, we also found that male circumcision was associated with a reduced risk of both cervical HPV and cervical cancer in the female partner. As shown in Figure 2, among monogamous women, circumcision status of the husband was associated with a reduced risk of cervical cancer, particularly and most strongly among women whose male consorts had engaged in high-risk sexual behaviours as measured by an early age at first sexual intercourse, a high lifetime number of sexual partners, and ever having sexual intercourse with women who were prostitutes. Furthermore, as shown in Figure 2, the use of a computed sexual behaviour risk index revealed a statistically significant dose-response relationship between increasing husband's sexual behaviour risk and wife's cervical cancer risk reduction linked to the husband's circumcision status. Concerning the effect of age at circumcision in this study, a somewhat greater protective effect for penile HPV, cervical HPV and cervical cancer was observed when the procedure was done after puberty or after sexual debut as compared to that performed at younger ages. However, the difference in the effects did not reach statistical significance.

Our finding that male circumcision may reduce the risk of cervical cancer in female sex partners is highly plausible for several quantitative reasons derived from further data analyses of the same IARC case-control study. ${ }^{10}$ First, because circumcision was found to be associated with a significant reduction in the risk of penile HPV infection; second, because penile HPV was also associated with a four-fold increased risk of cervical

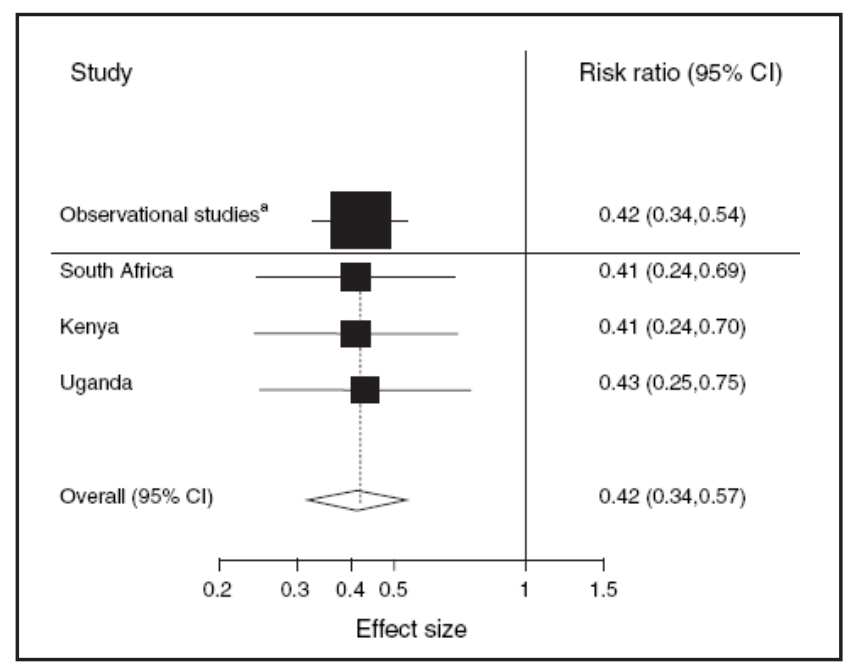

Figure 3 Meta-analysis for the association between male circumcision and HIV infection from data from three randomised controlled intervention trials of male circumcision in Africa and from 15 observational studies that adjusted for potential confounders. ${ }^{1}$ Figure reproduced, with permission, from: Weiss HA, Halperin D, Bailey RC, Hayes RJ, Schmid G, Hankins CA. Male circumcision for HIV prevention: from evidence to action? AIDS 2008; 22: 567-574. (C) Lippincott Williams \& Wilkins 
HPV infection in the female partner; and finally, because cervical HPV infection was also associated with a 77-fold increase in the risk of cervical cancer. Thus, within the same study data we consistently found strong associations in the three key steps occurring from male circumcision to cervical cancer development: (1) male circumcision reducing the risk of penile HPV, (2) penile HPV increasing the risk of cervical HPV and (3) cervical HPV increasing the risk of cervical cancer. Thus, circumcision can be considered an important co-factor in the natural history of HPV infection, since it may influence the risk of HPV acquisition and transmission as well as of cervical cancer development.

In conclusion, this IARC study proves strong epidemiological evidence that male circumcision is associated with a reduced risk of genital HPV infection in men and with a reduced risk of cervical cancer in women, notably among women with high-risk partners.

\section{From evidence to intervention}

The inverse associations found between male circumcision, HPV and cervical cancer are consistent with the current rapidly accumulating scientific evidence associating adult male circumcision with a reduced risk of acquiring and transmitting a number of STIs, ${ }^{11}$ in particular, the strong evidence from $\mathrm{RCTs}^{2-4}$ showing that male circumcision may substantially reduce the risk of HIV infection (Figure 3 ). ${ }^{1}$

Taking into account the overall evidence of the protective role of male circumcision on HIV, HPV and other STIs, male circumcision could be regarded as a potential one-time, single, affordable and safe global preventative option, acting like a global vaccine to reduce the burden of a number of STI-related diseases. Male circumcision should in particular be considered in adult men in high-risk countries that cannot afford the implementation of primary and secondary prevention strategies to control HIV, HPV and HPV-related cancers both in men and women.

Most routine circumcision procedures in developed countries occur almost exclusively in infancy or early childhood. A very small percentage of men require or request the procedure as adults. In contrast, in the clinical trials of male circumcision and HIV, the procedure is performed during adulthood. Thus, it remains to be better assessed whether infant circumcision also may exert longterm protective effects for HIV and HPV during adulthood, many years after the removal of the prepuce occurs.

In the context of the urgent need for initiatives to prevent AIDS and cervical cancer, the conclusive evidence that male circumcision reduces the risk of HIV and HPV acquisition is both promising and challenging. The evidence is solidly available and further data from intervention RCTs on circumcision and HPV should be available shortly. The challenge now is translating this evidence into public health policy: a complex task that will need to be context specific. The fact that the World Health Organization (WHO) and the Joint United Nations Programme on HIV/AIDS (UNAIDS) are endorsing the introduction or expansion of male circumcision programmes for HIV prevention should increase the demand for safe adult male circumcision services in populations with a high STI-related disease burden. ${ }^{1}$ International funding agencies such as the US President's Emergency Plan For AIDS Research (PEPFAR), the Agence Nationale de Recherche sur la SIDA (ANRS), the Bill and Melinda Gates Foundation and the US National
Institutes of Health are also supporting this initiative with further operational research. The scaling-up of the procedure taking into account its feasibility, cultural acceptability and the local epidemiology of HPV and HIV may result in a substantial reduction of the burden of both AIDS and cervical cancer in years to come. Close monitoring and evaluation of these programmes will certainly be needed to ensure that the procedure is well implemented and accompanied by counselling and follow-up to demonstrate that the intervention brings benefit and no harm.

\section{Statements on funding and competing interests}

Funding The work was partially supported by grants from the European Community [Cl $1-0371-\mathrm{F}(\mathrm{CD})]$; the Fondo de Investigaciones Sanitarias (FIS), Spain (86/753, 87/1513, 88/2049, 90/0901, 95/0955, 01/1237, 01/1236, and BAE 01/5013); Programa Interministerial de Investigación y Desarrollo, Spain (SAF 96/0323); Spanish public grants from the Instituto de Salud Carlos III (grants FIS PI030240, FIS PI061246, RCESP C03/09, RTICESP C03/10, RTIC RD06/0020/0095 and CIBERESP), from the Agència de Gestió d'Ajuts Universitaris i de Recerca (AGAUR 2005SGR 00695), and from the Marató de TV3 Foundation (051530), all of which had no role in the data collection, analysis or interpretation of the results.

Competing interests The authors have received educational, research or travel grants from the pharmaceutical industry as indicated: F Xavier Bosch - GlaxoSmithKline, Merck Sharp \& Dohme, Sanofi Pasteur MSD; Ginesa Albero - GlaxoSmithKline; Xavier Castellsagué - GlaxoSmithKline, Merck Sharp \& Dohme, Sanofi Pasteur MSD.

\section{References}

1 Weiss HA, Halperin D, Bailey RC, Hayes RJ, Schmid G, Hankins CA. Male circumcision for HIV prevention: from evidence to action? AIDS 2008; 22: 567-574.

2 Auvert B, Taljaard D, Lagarde E, Sobngwi-Tambekou J, Sitta $\mathrm{R}$, Puren A. Randomized, controlled intervention trial of male circumcision for reduction of HIV infection risk: the ANRS 1265 Trial. PLoS Med 2005; 2: e298.

3 Bailey RC, Moses S, Parker CB, Agot K, Maclean I, Krieger $\mathrm{JN}$, et al. Male circumcision for HIV prevention in young men in Kisumu, Kenya: a randomised controlled trial. Lancet 2007; 369: 643-656.

4 Gray RH, Kigozi G, Serwadda D, Makumbi F, Watya S, Nalugoda F, et al. Male circumcision for HIV prevention in men in Rakai, Uganda: a randomised trial. Lancet 2007; 369: 657-666.

5 Castellsague X, Albero G, Cleries R, Nielson C, Giuliano A, Bosch FX. HPV and circumcision: a biased, inaccurate and misleading meta-analysis. J Infect 2007; 55: 91-93.

6 Vaccarella S, Lazcano-Ponce E, Castro-Garduno JA, CruzValdez A, Diaz V, Schiavon R, et al. Prevalence and determinants of human papillomavirus infection in men attending vasectomy clinics in Mexico. Int J Cancer 2006; 119: 1934-1939.

7 Nielson CM, Flores R, Harris RB, Abrahamsen M, Papenfuss $M R$, Dunne EF, et al. Human papillomavirus prevalence and type distribution in male anogenital sites and semen. Cancer Epidemiol Biomarkers Prev 2007; 16: 1107-1114.

8 Partridge JM, Hughes JP, Feng Q, Winer RL, Weaver BA, Xi LF, et al. Genital human papillomavirus infection in men: incidence and risk factors in a cohort of university students. $J$ Infect Dis 2007; 196: 1128-1136.

9 Castellsague X, Albero G, Cleries R, Nielson C, Giuliano A, Bosch FX. Male circumcision and penile HPV: an updated meta-analysis. 24th International Papillomavirus Conference and Clinical Workshop, Beijing, China, 3-9 November 2007; Abstract 8B: 117

10 Castellsague X, Bosch FX, Munoz N, Meijer CJ, Shah KV, de Sanjose $S$, et al. Male circumcision, penile human papillomavirus infection, and cervical cancer in female partners. N Engl J Med 2002; 346: 1105-1112.

11 Castellsague X, Peeling RW, Franceschi S, de Sanjose S, Smith JS, Albero G, et al. Chlamydia trachomatis infection in female partners of circumcised and uncircumcised adult men. Am J Epidemiol 2005; 162: 907-916. 